\title{
Double-Core GMI Current Sensor
}

\author{
Michal Malátek ${ }^{1}$, Pavel Ripka ${ }^{1}$, and Ludĕk Kraus ${ }^{2}$ \\ ${ }^{1}$ Faculty of Electrical Engineering, Czech Technical University, 16627 Prague, Czech Republic \\ ${ }^{2}$ Institute of Physics, Academy of Science of the Czech Republic, 18221 Prague, Czech Republic
}

\begin{abstract}
A novel design of the giant magnetoimpedance contactless current sensor is presented. A double-core structure is used in order to improve the temperature stability. The temperature coefficient of sensitivity and offset drift are reduced to one-half compared to the single-core sensor. The linearity error decreases by a factor of three. Further utilization of an ac biasing (up to $200 \mathrm{~Hz}$ ) of the double-core sensor suppresses the temperature offset drift by a factor of 30 (down to $0.6 \mathrm{~mA} / \mathrm{K}$ ) and increases the open-loop linearity to $0.5 \%$ for the 2-A range.
\end{abstract}

Index Terms-Amorphous ribbon, current sensor, giant magnetoimpedance (GMI), temperature stability.

\section{INTRODUCTION}

G IANT magnetoimpedance (GMI) is an intensively studied phenomenon that can be utilized in various sensors of a magnetic field and mechanical variables. For current sensing, the GMI effect was used first by Prieto et al. [1]. The ring shape of the GMI current sensor was proposed by Ovari et al. [2]. A GMI current sensor was also patented [3]. A current sensor based on an asymmetrical GMI effect was reported in [4]. Although the GMI phenomenon has been thoroughly investigated [5], not much attention was paid to the temperature properties of GMI sensors in the industrial temperature range $\left(-20{ }^{\circ} \mathrm{C}\right.$ to $+80^{\circ} \mathrm{C}$ ). Recently, we have analyzed the performance of a contactless GMI current sensor [6]. We believe that besides the perming effect, the temperature dependence is the main factor limiting the practical application of GMI sensors.

As we have shown with Co-rich amorphous ribbon [7], there is a strong temperature dependence of circular permeability, which causes variations of sensitivity and the offset of GMI sensors. The main disadvantage of the first GMI current sensor, which we developed, was the high-temperature drift of the offset [7], [8]. Here, we present a novel design of a double-core GMI current sensor with reduced temperature instability and improved linearity.

Our sensor uses a symmetrical GMI effect. The even characteristics is achieved by an ac bias. We believe that this solution can be more stable than the one using asymmetrical GMI characteristics [5]. There, the asymmetry is achieved by surface crystallization as well as internal stress, which are supposed to be temperature dependent and unstable in time.

\section{SENSOR STRUCTURE}

The double-core sensor (Fig. 1) consists of two 31-mm diameter ring cores each wound by 52 -cm-long $\mathrm{Co}_{67} \mathrm{Fe}_{4} \mathrm{Cr}_{7} \mathrm{Si}_{8} \mathrm{~B}_{14}$ amorphous strips. In order to optimize the MI characteristics, the ribbon was annealed for $10 \mathrm{~min}$ at $390{ }^{\circ} \mathrm{C}$ under an axial field of $2400 \mathrm{kA} / \mathrm{m}$. The magnetic strips are wound on ceramic rings (Fig. 2), and these cores are electrically connected in series and excited by RF current. Two toroidal 260-turn antiserially

Digital Object Identifier 10.1109/TMAG.2005.854810

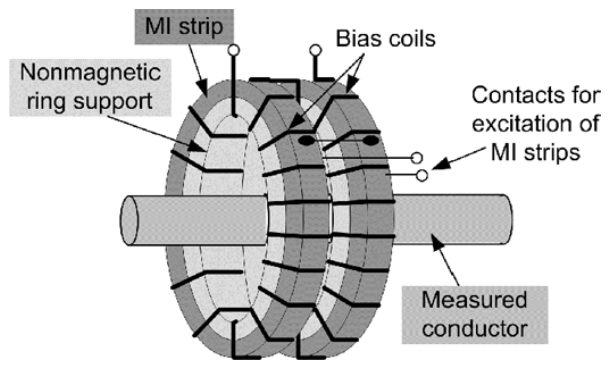

Fig. 1. Design of double-core sensor.

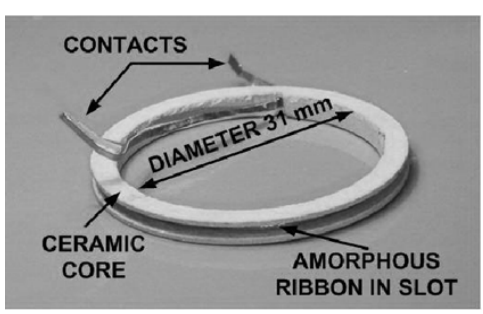

Fig. 2. Photo of single-core sensor without bias coil.

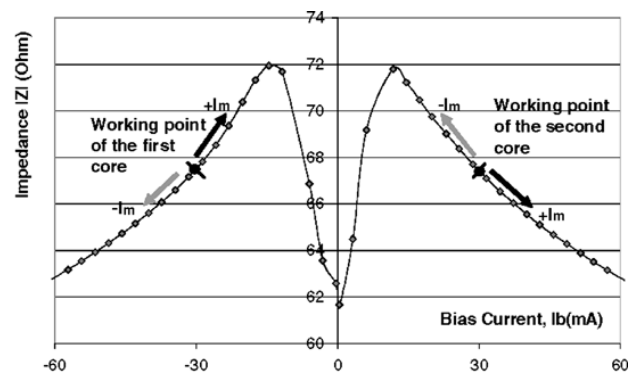

Fig. 3. Working characteristic of doube-core sensor.

connected bias coils are wound around the rings. The bias coils shift the working points of the cores to the linear part of magnetoimpedance characteristics (Fig. 3). A circular magnetic field of the measured current $I_{m}$ is superimposed to the bias fields and the total magnetization decreases in one core and increases in the other. The voltage drops over the two cores are amplified and then subtracted by a differential amplifier.

Assuming that the impedances of both cores have similar temperature dependencies, one can expect that the subtraction of the voltage drops would partly compensate the temperature sensitivity of the offset. The characteristics at the working point of 


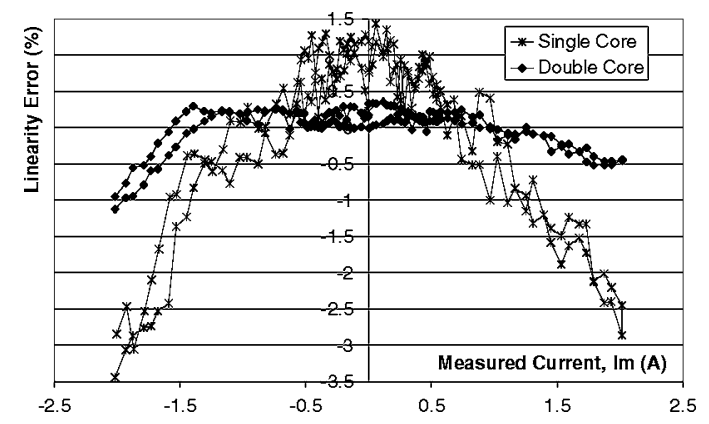

Fig. 4. Linearity error of single and double core sensor structure.

TABLE I

LIST OF PARAMETERS

\begin{tabular}{lcc}
\hline \hline & $\begin{array}{c}\text { Single } \\
\text { core }\end{array}$ & $\begin{array}{c}\text { Double } \\
\text { core }\end{array}$ \\
\hline Offset at room temperature $(\mathrm{mA})$ & - & 100 \\
Linearity error in the range $\pm 2 \mathrm{~A}(\% \mathrm{FSR})$ & 3.44 & 1.13 \\
Temperature coefficient of sensitivity $\left(\% /{ }^{\circ} \mathrm{C}\right)$ & 0.15 & 0.08 \\
Temperature coefficient of offset $\left(\mathrm{mA} /{ }^{\circ} \mathrm{C}\right)$ & 32.16 & 19.21 \\
\hline \hline
\end{tabular}

Coming from a principle of the single-core sensor, it has non-zero offset, which is several times higher than measurement range.

each core is nonlinear; the slopes of the first and the second core are increasing and decreasing, respectively, with positive current. Due to this fact, the linearity of the double-core sensor is improved, supposing that the shapes of both characteristics are similar.

\section{MEASUREMENTS AND Results}

The sensor was driven at a $1-\mathrm{MHz} / 5-\mathrm{mA}_{\mathrm{RMS}}$ sinewave signal and the bias current $I_{b}$ was $30 \mathrm{~mA}$, corresponding to a bias field of $80 \mathrm{~A} / \mathrm{m}$. Each core was first tested separately, in the same setup as in [8] (the single-core structure). Afterwards, the cores were connected together to form the double-core sensor.

The measurements were accomplished with the sensors placed in a magnetically shielded temperature chamber with an accessible temperature span between $-20^{\circ} \mathrm{C}$ and $+80^{\circ} \mathrm{C}$. The sensor responses to the measured current $I_{X}$ in the range of $\pm 2 \mathrm{~A}$ were tested at different temperatures.

The double-core sensor shows better linearity (Fig. 4) and smaller temperature offset drift compared to the single-core sensor. On the other hand, manufacturing a double-core sensor is more difficult, since suitable pairs of magnetic cores with similar material properties should be selected. Because the samples coming even from the same ribbon can have different magnetoimpedance characteristics, an additional correction by detection electronics is usually needed.

The difference between the single- and the double-core sensors is illustrated in Table I. The offset value of the single-core sensor is given by its bias field and it exceeds several times the full-scale range. The frequency bandwidth of both structures is expected to be dependent on the frequency of the driving signal, and maximal detected frequency should be lower than half of the driving frequency.

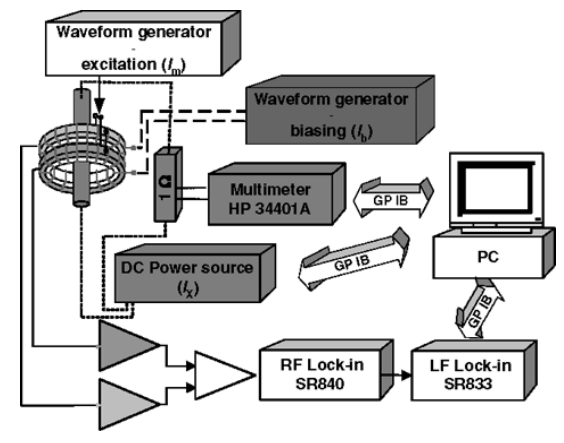

Fig. 5. Measurement setup for ac-biased GMI current sensor.

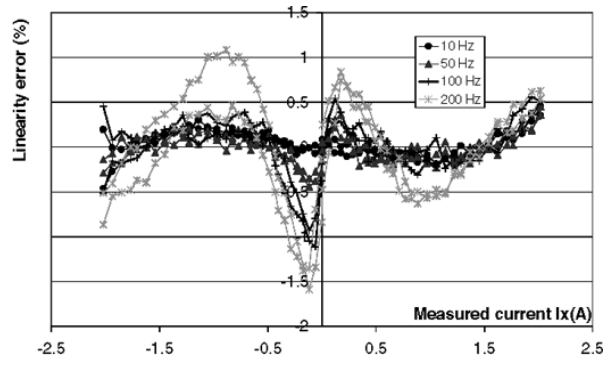

Fig. 6. Linearity errors of sensor at different bias frequency.

\section{AC-BIASED SENSOR}

Additional sensor development was directed to the application of an alternating bias field to the cores. It has been proved that by implementation of the ac-bias field the linearity error and the offset of the sensor could be further reduced [9]. This method, however, substantially reduces the maximum measurement frequency range. So, it is suitable only for low frequency and dc applications. The other drawback is a requirement of more complicated signal conditioning.

The sensor was biased by $30-\mathrm{mA}_{\mathrm{RMS}}$ squarewave signal of frequencies from 10 to $200 \mathrm{~Hz}$. Two waveform generators were used in order to operate the sensor: one for the excitation of GMI strips and the second for the ac supplying of the bias coils. Two lock-in amplifiers connected in cascade were used for the output signal demodulation. The measurement setup is shown in Fig. 5. The sensor underwent the same measurement procedure as in the case of the dc biasing.

Measured results confirmed an improvement of the linearity using the ac-bias technique. Fig. 6 shows the linearity error of the sensor at different bias frequencies. The best results were obtained at the ac bias of 10 and $50 \mathrm{~Hz}$, respectively (circles and triangles), where linearity error of the sensor in the open loop does not exceed $0.5 \%$ of the full-scale range. With increasing frequency, an increase of nonlinearity in near-zero values of the $I_{x}$ was observed. This correlation is probably caused by a spurious signal associated with current transients in the bias coil.

The offset of ac-biased sensor $(10 \mathrm{~Hz})$ varied between 0 and $55 \mathrm{~mA}$ for the temperature sweeping from $-20{ }^{\circ} \mathrm{C}$ to $72{ }^{\circ} \mathrm{C}$, which results in a very small temperature offset drift coefficient of $0.6 \mathrm{~mA} /{ }^{\circ} \mathrm{C}$. On the other hand, the temperature coefficient of the sensitivity shows stronger temperature influence compared even to the single-core sensor. The temperature influence can be seen in Fig. 7, where the sensor responses to the measured current $I_{X}$ are shown. For bias frequency of $10 \mathrm{~Hz}$, this coefficient 


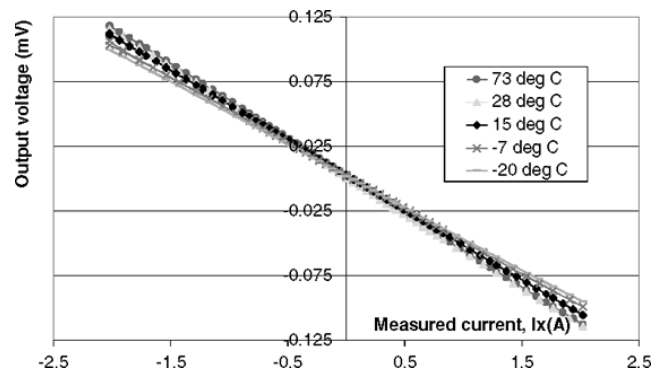

Fig. 7. AC-biased sensor responses at different temperatures.

TABLE II

PARAMETERS OF AC-BIASED SENSOR

\begin{tabular}{lccc}
\hline \hline \multicolumn{1}{c}{ Bias frequency $(\mathrm{Hz})$} & 10 & 50 & 100 \\
\hline Offset at room temperature $(\mathrm{mA})$ & 35 & 50 & 68 \\
Linearity error in the range $\pm 2 \mathrm{~A}(\% \mathrm{FSR})$ & 0.49 & 0.45 & 1.1 \\
Temperature coefficient of sensitivity $\left(\% /{ }^{\circ} \mathrm{C}\right)$ & 0.18 & 0.18 & 0.19 \\
Temperature coefficient of offset $\left(\mathrm{mA} /{ }^{\circ} \mathrm{C}\right)$ & 0.6 & 0.6 & 0.7 \\
\hline \hline
\end{tabular}

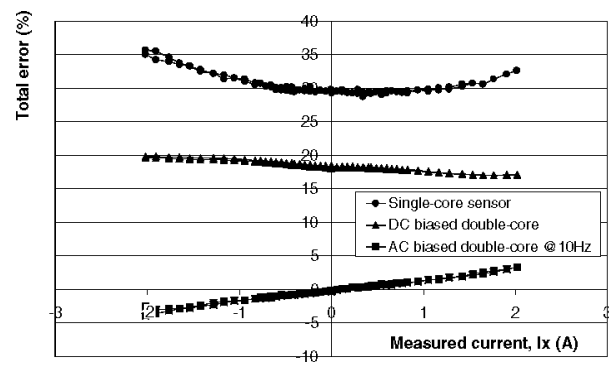

Fig. 8. Worst case of total error within temperature span between $-20{ }^{\circ} \mathrm{C}$ to $+80^{\circ} \mathrm{C}$. Deviations are referred to sensor responses at room temperature. Total error covers temperature offset drift, sensitivity tempco, and nonlinearity of sensor.

reaches a value of $0.18 \% /{ }^{\circ} \mathrm{C}$. It should be noted, however, that the temperature coefficient of sensitivity can be substantially reduced by using the feedback. The other parameters are summarized in Table II as well as the parameters at $50-$ and $100-\mathrm{Hz}$ biasing.

In Fig. 8, there is the comparison of the worst case of deviation of the three setups from their reference response at room temperature. The deviation is a sum of three contributors: the linearity error, the temperature offset drift, and the temperature coefficient of the sensitivity. The largest difference occurred at $-20{ }^{\circ} \mathrm{C}$ for all three setups. One can easily distinguish the offset drift as a major contributor to the deviation in case of the single-core and the dc-biased double-core sensor.

\section{CONCLUSION}

The major drawback of GMI sensors, using Co-rich amorphous materials or crystalline materials like permalloys, is their remarkable temperature instability, the main origin of which is the temperature dependence of circular permeability of the ferromagnetic conductor. Even though the parameters of the sensor, which we have achieved, are still far from industrial requirements on precise current sensors (an example of commercial fluxgate current sensor with 40-A range has accuracy better than $0.5 \%$ and temperature drift $<30 \mu \mathrm{A}$ in temperature span $-20{ }^{\circ} \mathrm{C}$ to $+70{ }^{\circ} \mathrm{C}$ ), we have shown that appropriate sensor design in connection with a suitable signal conditioning could noticeably improve the parameters.

When a large working temperature range and accuracy are required, the dual-core GMI sensor is probably the best solution. Then, a very careful matching of the core pairs and/or precise adjustment of the detection circuitry (which must be in close relation with parameters of particular cores) are necessary. The serial production of such sensors would be, however, very expensive. This is currently the basic limitation of the double-core sensor, which calls for improved technologies providing more homogenous material properties.

Employment of the ac-bias scheme complicates the electronics and limits the bandwidth, but its result is very effective suppression of the offset temperature drift by the factor of 30 (from 19 to $0.6 \mathrm{~mA} /{ }^{\circ} \mathrm{C}$ ).

In a small temperature span, the accuracy is limited by the linearity, which can be solved by the double-core structure of the sensor with dc or ac bias and/or by a feedback compensation. This technique would also suppress the temperature coefficient of the sensitivity.

\section{ACKNOWLEDGMENT}

This work was supported by the Ministry of Education, Youth and Sport, Czech Republic, under Project KONTAKT Me696.

\section{REFERENCES}

[1] M. J. Prieto, A. M. Pernia, J. M. Lopera, F. Nuno, F. Linera, and J. Diaz, "Amorphous materials application in current sensors," Revista Espanola de Electronica, no. 520, pp. 70-74, 1998.

[2] T.-A. Óvári, H. Chiriac, C. S. Marinescu, F. J. Castaño, M. Vázquez, and A. Hernando, "Magneto-impedance response in ring shaped amorphous wires," Sens. Actuators A, Phys., vol. 91, no. 1-2, pp. 207-209, 2001.

[3] R. Valenzuela, M. Vazquez, A. Hernando, and J. J. Freijo, Patent P95 502081

[4] Y. W. Rheem, C. G. Kim, C. O. Kim, and S. S. Yoon, "Current sensor application of asymmetric giant magnetoimpedance in amorphous materials," Sens. Actuators A, Phys., vol. 106, no. 1-3, pp. 19-21, 2003.

[5] Handbook of Magnetic Materials, vol. 15, K. H. J. Buschow, Ed., NorthHolland, Amsterdam, The Netherlands, 2003, pp. 497-563.

[6] P. Ripka, "Current sensors using magnetic materials," J. Optoelectron. Advanced Mater, vol. 6, no. 2, pp. 587-592, 2004.

[7] M. Malátek, L. Kraus, and P. Ripka, "Temperature drift of offset of MI sensors," in Proc. EMSA 2004, vol. M-P.14, Cardiff, U.K..

[8] M. Malátek, A. Platil, and P. Ripka, "GMI current sensor," in Eurosens. XVII. Guimaraes, 2003, pp. 418-419.

[9] M. Malátek, A. Platil, A. Cerman, and P. Ripka, "Permalloy GMI sensor," in Proc. Magnetic Measurements Conf., Bratislava, 2002, p. 109.

Manuscript received January 7, 2005. 